\title{
Uso de Internet e impulsividad en estudiantes mexicanos de secundaria y bachillerato
}

\author{
Janeth Contreras-Aburto', Paulina Beverido-Sustaeta², Xóchitl de San Jorge-Cárdenas², Betzaida Salas-García², \\ María Cristina Ortiz-León ${ }^{3}$
}

I Facultad de Medicina, Universidad Veracruzana, México

2 Instituto de Ciencias de la Salud, Universidad Veracruzana, México

${ }^{3}$ Instituto de Salud Pública, Universidad Veracruzana, México

\section{RESUMEN}

Introducción: la mayoría de los adolescentes usa Internet sin que esto repercuta en su salud; sin embargo, algunos pueden presentar pérdida de control sobre la manera en la que utilizan este medio, lo que conlleva a un uso problemático que puede derivar en adicción. Objetivo: identificar los tipos de uso de Internet en estudiantes de secundaria y bachillerato, sus posibles causas y su relación con la impulsividad. Método: se realizó un estudio observacional, transversal, analítico y ex post facto en una muestra estratificada de 123 estudiantes de secundaria y 125 de bachillerato, de entre 12 y 18 años de edad, inscritos en dos escuelas de Xalapa, Veracruz, México; se utilizó la prueba de Adicción a Internet de Young en español. Resultados: $83 \%$ de los estudiantes presentó control sobre el uso de Internet, 17\% mostró un uso problemático y no se encontró adicción a la red. El uso problemático fue mayor entre los estudiantes de secundaria (23\%), que entre los de bachillerato (10\%). La impulsividad no fue estadísticamente significativa para presentar un uso problemático, pero sí lo fueron indicadores como estar conectado más de seis horas y el empleo exclusivo de Internet para consultar y relacionarse en las redes sociales. Discusión y conclusiones: no se encontró una relación significativa entre impulsividad y uso problemático de Internet; sin embargo, los factores de exposición pueden explicar que el individuo disminuya el contacto con su entorno familiar y social. Los hallazgos de este trabajo pueden constituir una guía para futuras líneas de investigación que profundicen en el conocimiento de este problema.

Palabras claves: uso de Internet, adolescentes, factores de exposición, impulsividad.

\begin{abstract}
Introduction: most adolescents use the Internet without any impact on their health, however some people in this population group may present a loss of control over the use of it, which can lead to a problematic use and even an addiction to the Internet. Objective: to identify the types of Internet use in high school students, the possible causes and its relationship with impulsivity. Method: an observational, transversal, analytical and ex post facto study was performed in a stratified sample of 123 middle school students and 125 high school students aged 12 to 18 enrolled in two schools in Xalapa, Veracruz, Mexico, using the Internet Addiction Test by Young, in Spanish. Results: $83 \%$ of the students present control over the use of the Internet, $17 \%$ presented a problematic use and no Internet addiction was found. The problematic use was higher among middle school students $(23 \%)$ than among high school students (10\%). Impulsivity was not statistically significant related with a problematic use of Internet, but using it more than six hours and using it exclusively for social networks were positive indicators. Discussion and conclusions: there was no significant relationship between impulsivity and problematic use of the Internet; however, the associated risk factors may cause a decrease of students' family and social interaction. The findings of this work can be a guide for future lines of research that deepen the knowledge of this problem.
\end{abstract}

Keywords: use of internet, adolescents, risk factors, impulsivity.

\footnotetext{
Autor de correspondencia:

María Cristina Ortiz-León. Av. Doctor Luis Castelazzo Ayala S.N., col. Industrial Ánimas, C.P. 91190, Xalapa Enríquez, Veracruz, México. Correo electrónico: cortiz@uv.mx

Recibido: 5 de julio de 2017

Aceptado: 18 de septiembre de 2017

DOI: 10.28931/riiad.2017.2.02
} 


\section{INTRODUCCIÓN}

Durante los últimos años del siglo XX, el mundo participó en una profunda revolución tecnológica que trajo consigo nuevas prácticas de comunicación e interacción personal. La Internet introdujo a la sociedad a la era de la información y revolucionó las tecnologías de la información y la comunicación (TIC), impulsando la generación de dispositivos, redes y aplicaciones cuyo impacto en los comportamientos sociales -en particular en el de los jóvenes- apenas empieza a ser evaluado (Ontiveros, 2015; Pérez del Río, 2014).

Sin duda, la Internet aporta numerosos beneficios, pues brinda oportunidades únicas para la comunicación, el entretenimiento y la educación (Castellana Rosell, Sánchez-Carbonell, Graner Jordana, \& Berauny Fargues, 2007); los grandes volúmenes de información disponibles en la red ayudan, además de a incrementar el conocimiento, a realizar transacciones comerciales y trámites administrativos. Se trata de una herramienta de comunicación que favorece el establecimiento de relaciones interpersonales y la participación en juegos, por lo que se ha transformado en un importante medio de esparcimiento (Cía, 2013) que sobrepasa las barreras geográficas, demográficas, socioeconómicas, culturales e ideológicas (Rial, Gómez, Braña, \& Varela, 2014).

Aunque los beneficios de la Internet son múltiples y diversos, hay personas, especialmente jóvenes y adolescentes, que se muestran incapaces de controlar el tiempo que navegan en ella y de utilizar esta importante herramienta para mejorar sus conocimientos, habilidades y valores sin afectar de forma negativa sus vínculos personales y familiares, su rendimiento académico o su trabajo (Suriá, 2015).

En el ámbito científico no hay consenso sobre si se trata de una nueva adicción y menos aún sobre los criterios para su diagnóstico, evaluación y tratamiento, de manera que lo mismo se alude a la adicción a la Internet (Young, 1998), que al desorden de adicción a Internet (IAD, por sus siglas en inglés; Goldberg, 1995), al uso compulsivo de Internet (Greenfield, 1999) o al uso patológico de Internet (Koran, Faber, Aboujaoude, Large, \& Serpe, 2006). Es preciso señalar que el Manual Diagnóstico y Estadístico de los Trastornos Mentales DSM-5 (American Psychiatric Association, 2014) no ha reconocido este problema como una patología.

Es importante considerar que cuando se habla de adicción, se reconoce la presencia de dos factores: la pérdida de control y la dependencia. Se entiende a la primera como la incapacidad de parar una vez iniciada una conducta; y a la segunda, como la necesidad de realizarla y de ajustar a ella la vida cotidiana (Oliva Delgado et al., 2012). Para efectos de este trabajo, se define como Adic- ción a la Internet (Al), a las prácticas que activan en el cerebro sistemas de recompensa y que provocan síntomas conductuales similares a los que derivan del abuso de sustancias psicoactivas o de conductas no relacionadas con sustancias, como el uso de los videojuegos.

De acuerdo con algunos autores, se trata de una afición patológica que genera dependencia y resta libertad al ser humano, al estrechar su campo de conciencia y restringir sus intereses; tiene manifestaciones sintomáticas en lo cognitivo, lo conductual y lo fisiológico, y puede llegar a interferir de manera grave en la vida cotidiana de las personas, a nivel familiar, escolar, social o de salud (Echeburúa \& de Corral, 1994; Young, 1998).

De acuerdo con el Centro Pedagógico Paideia, se observan indicios de empleo problemático de Internet -preámbulo de la Al-, cuando el incremento de su uso produce una interferencia grave en la vida cotidiana, y la persona, por lo general adolescente, pierde interés en la realización de otras actividades. Según CaldeviIla (2010), el uso problemático de la red tiene diversas manifestaciones: atención que se coloca de manera simultánea en diversos asuntos y dispositivos electrónicos; reducción de la productividad laboral y escolar; entronización de la individualidad; degradación del lenguaje; necesidad de respuestas inmediatas, que rebasa el espacio de Internet; adopción de conductas riesgosas y ajenas al comportamiento cara a cara; y mayor posibilidad de ser objeto o autor de conductas delictivas o fraudulentas. Otros autores señalan que cuando se registra un consumo problemático es posible observar alteraciones psicológicas que afectan el estado de ánimo, así como un aumento del aislamiento social y el paradójico deterioro de las relaciones afectivas más cercanas (Castellana Rosell et al., 2007; Cía, 2013; García del Castillo, 2013; Muñoz-Rivas, Navarro, \& Ortega, 2003; Oliva Delgado et al., 2012).

Países de Europa y Asia están mostrando verdadero interés en este tema, lo que ha derivado en evaluaciones que estiman una prevalencia del uso problemático de Internet, entre los adolescentes y adultos jóvenes, que va de 1 a $9 \%$ en Europa, de 1 a 12\% en Oriente Medio, de 2 a 18\% en Asia (Christakis, 2010) y de 0 a 26.3\% en Estados Unidos (An et al., 2014). En Latinoamérica no existen cifras sobre esta prevalencia, a pesar de que investigadores de algunos países como Colombia (Puerta-Cortés \& Carbonell, 2013), México (Beverido-Sustaeta, Camarena-Matús, Cruz-Juárez, \& Ortiz-León, 2016; Gámez Guadix \& Villa George, 2015; Hernández Contreras, Ponce Rojo, \& Castañeda Barajas, 2015; Méndez, Zepeda, \& Galván, 2014) y Perú (Lam-Figueroa et al., 2011) han empezado a ocuparse de esta problemática.

Además de ser el grupo poblacional que más utiliza Internet, los adolescentes son especialmente vulnera- 
bles a desarrollar un uso problemático y Al por diversas razones, entre las que destacan las siguientes: la cantidad de dispositivos que permiten el acceso a la red; los servicios y aplicaciones que ofrece; la rapidez para obtener contenidos de interés; la disponibilidad; y la posibilidad de conservar, sin consecuencias, el anonimato, lo que permite y se ajusta a la búsqueda de sensaciones, nuevas experiencias y desinhibición, propia de este grupo poblacional (Oliva Delgado et al., 2012).

Otros estudios Ilaman la atención sobre el aumento en el riesgo de $\mathrm{Al}$ ante la presencia de problemas psiquiátricos como la depresión, el Trastorno por Déficit de Atención e Hiperactividad (TDAH) y la fobia social u hostilidad (Echeburúa \& de Corral, 2010). En particular, la impulsividad -con frecuencia ligada al TDAH- es un factor que se ha vinculado con comportamientos disfuncionales como el contacto temprano con drogas, la repetición del consumo y la progresión a la adicción (Pedrero Pérez, 2009).

Quienes no reconocen la existencia de la Al, se apoyan en la carencia de evaluaciones comparables en distintas poblaciones y, sobre todo, en la ausencia de instrumentos de diagnóstico consensuado para realizar estos estudios (Camelo, León, \& Salcedo, 2013). En respuesta, han empezado a surgir instrumentos que resultan de la adaptación de los criterios de adicción contenidos en el DSM-5 para dependencia de sustancias y trastornos de juego patológico: Young (1998) diseñó la primera versión de la Prueba de Adicción a Internet (PAI), un cuestionario aplicado a diferentes muestras de sujetos, con el que se pueden descubrir los numerosos síntomas asociados con este problema; si bien existen otros instrumentos para medir esta variable, esta escala ha sido la más utilizada en entornos clínicos y de investigación. Aunque en Perú se utilizó la Escala de Adicción a Internet de Lima (EAIL) para evaluar la Al en población adolescente -un instrumento que presenta limitaciones debido a que no define el punto de corte para diagnosticar la adicción (Lam-Figueroa et al., 2011)-, los estudios sobre el tema realizados en Colombia (Puerta-Cortés \& Carbonell, 2013) y México (Hernández Contreras, Ponce Rojo, \& Castañeda Barajas, 2015; Méndez et al., 2014; Beverido-Sustaeta et al., 2016) emplearon la versión en español del Internet Addiction Test (IAT), desarrollado por Young.

En lo que corresponde a la revisión de los antecedentes de las variables que abarca el presente trabajo, como la impulsividad, es importante recordar que el DSM-IV incluyó a las adicciones psicológicas dentro de los trastornos del control de impulsos. En el mismo tenor, se encontró un estudio realizado en 2014 con 1068 estudiantes de medicina de tres universidades del norte de China, que relacionó la Al con algunas características psicológicas como impulsividad, sentido de vida y autoestima; la investigación concluyó que existe una relación estadísticamente significativa entre un mayor rasgo de impulsividad y el incremento de la tendencia a presentar adicción a Internet. Además, encontró que factores como el sentido de vida y la autoestima positivos pueden funcionar como protectores contra la adicción a Internet en personas muy impulsivas (Zhang et al., 2015). En México, sólo se identificó un estudio que evaluó el papel de la depresión y la impulsividad en adolescentes mexicanos, y mostró una relación estadísticamente significativa entre las variables de interés (Gámez Guadix \& Villa George, 2015).

De acuerdo con la Encuesta Nacional sobre Disponibilidad y Uso de Tecnologías de la Información en los hogares (ENDUTIH), elaborada por el Instituto Nacional de Estadística y Geografía (INEGI, 2015), en México, alrededor de 62.4 millones de personas de seis años y más son usuarias de Internet -sobre todo en el medio urbano- y 12.8 millones de hogares mexicanos (39.2\%) cuentan con conexión a la red. La primera de estas estadísticas es importante porque representa a poco más de la mitad de la población (57.4\%) del país y porque se observa un incremento de 4.8 puntos porcentuales, respecto del año anterior (Instituto Nacional de Estadística y Geografía, 2015).

Considerando lo antes descrito y el alto porcentaje de adolescentes conectados a la red en nuestro país, el objetivo de este trabajo es identificar los tipos de uso de Internet, sus posibles causas y su relación con la impulsividad, en estudiantes de dos escuelas de México, una de secundaria y otra de bachillerato.

\section{MÉTODO}

Se desarrolló un estudio observacional, transversal, analítico, ex post facto, para establecer la asociación entre el uso de Internet y los posibles factores que lo condicionan en un momento determinado, sin manipular las variables de interés. El estudio se llevó a cabo en dos escuelas públicas, una secundaria y una preparatoria, de la ciudad de Xalapa, Veracruz, México, y se utilizó un muestreo probabilístico estratificado, dependiente de los grados y el nivel de escolaridad que cursaban los adolescentes. Los criterios de inclusión fueron estar inscritos en el período lectivo 2015-2016 y tener entre 12 y 18 años; se excluyeron aquellos alumnos que tuvieran algún diagnóstico de trastorno psicológico o psiquiátrico. Se calculó un tamaño de muestra proporcional al número total de alumnos en cada grado escolar del nivel respectivo, por lo que participaron 248 del total de 727 alumnos (34.11\%). La recolección de la información se realizó entre enero y abril del 2016. 
La variable dependiente fue el tipo de uso de Internet y las independientes fueron sexo, edad, nivel de escolaridad, impulsividad y aspectos relacionados con el empleo de esta herramienta. Se utilizó un instrumento para la recolección de la información, resultante de la integración de un cuestionario y dos escalas. El cuestionario se elaboró con el propósito de recabar datos sociodemográficos (edad, sexo, grado y nivel de escolaridad) y de uso de Internet (medio, lugar, tiempo de acceso y actividades realizadas).

Aunque se conocen alrededor de 18 escalas, se eligió la Prueba de Adicción a Internet (IAT, por sus siglas en inglés) debido a que fue validada en español por Puerta-Cortés y Carbonell (2013) y a que es usada de manera frecuente en este tipo de estudios, lo que permitió realizar comparaciones; este instrumento de tamizaje fue desarrollado a partir de una adaptación de los criterios utilizados para evaluar juego patológico y consta de 20 ítems valorados en una escala Likert, cuyos rangos de puntuación van de 20 a 100 y clasifican el tipo de uso de Internet en:

1) Control sobre el uso de Internet (20 a 49 puntos).

2) Uso problemático de Internet (50 a 79 puntos).

3) Uso adictivo o Al (Más de 80 puntos).

La segunda escala, también de tamizaje, fue la Escala de Impulsividad de Barratt versión $11 \mathrm{~A}$, adaptada a población adolescente y traducida al español; está compuesta por 30 ítems que se agrupan en tres subescalas: impulsividad cognitiva, conformada por 8 ítems; impulsividad motora, compuesta de 10 ítems; e impulsividad no planeada, con 12 ítems. El rango de variación de la escala es de 30 a 120 y el punto de corte para considerar impulsividad fue de 73 (Malloy-Diniz et al., 2015; Martínez-Loredo, Fernández-Hermida, Fernández-Artamendi, Carballo, \& García-Rodríguez, 2015).

Una vez integrado un instrumento de 50 ítems, se procedió a realizar una prueba piloto con el propósito de detectar fallas que evitaran la cabal comprensión de las preguntas; para ello se seleccionaron, a conveniencia, 30 estudiantes: 15 de secundaria y 15 de bachillerato, en sedes distintas a donde se realizó el estudio. Como resultado de esta prueba, se hicieron modificaciones de sintaxis en algunas preguntas para adecuarlas al contexto de la población.

A nivel estadístico, se realizó un análisis descriptivo de las variables cualitativas, expresadas en porcentajes; mientras que para las variables numéricas, se usaron medidas de tendencia central y dispersión. La información se capturó y analizó utilizando el programa IBM SPSS Statistics 21. La relación entre el uso problemático de Internet y otras variables fue medida con la razón de momios (Odds Ratio $[O R]$ ), con sus respectivos intervalos de confianza de 95\%, obtenidos a través de la herramienta online OpenEpi. Se eligió la razón de momios, ya que permitió establecer de manera univariada la contribución de cada una de las variables consideradas en el estudio.

En cuanto a las consideraciones éticas, antes de aplicar el instrumento se sostuvo una entrevista con las autoridades escolares para explicarles los objetivos y los beneficios del estudio; asimismo, se garantizó la confidencialidad de los datos obtenidos y el anonimato de los participantes. Tras esto, se acordó el calendario para la aplicación del instrumento y se solicitó la lista de asistencia de cada grado por nivel, para realizar la selección aleatoria de los alumnos.

En consideración de que la mayor parte de los estudiantes de secundaria y bachillerato son menores de edad, además del consentimiento de las autoridades educativas, se solicitó a los participantes la entrega de una carta de consentimiento informado -elaborada por el equipo investigador-firmada por el padre o tutor.

Antes de la aplicación de la prueba, los estudiantes fueron informados sobre los objetivos del estudio, sobre el carácter voluntario y anónimo de su participación y sobre el uso que se daría a la información resultante $y$, aunque no hubo asentimiento escrito, los estudiantes expresaron de manera verbal su interés por participar en el estudio.

\section{RESULTADOS}

De los 248 alumnos participantes, 123 estudiaban secundaria y 125 bachillerato (44\% hombres y 56\% mujeres); la edad promedio fue de 14.8 años $(D E=1.8)$. Al comparar los resultados entre los estudiantes de secundaria y bachillerato, las variables que fueron estadísticamente significativas fueron edad, uso de Internet, modo de acceso, tiempo de uso, uso de Internet para conectarse a redes sociales, uso de Internet como entretenimiento e impulsividad (Tabla 1).

El estudio mostró que $83 \%$ de los estudiantes presentó control del uso de Internet; y 17\%, un uso problemático -mayor en estudiantes de secundaria que en los de bachillerato-; no se encontró AI. 72\% de los participantes reportó que se conecta a través del teléfono móvil, con una proporción mayor entre estudiantes de bachillerato (82\%) que entre los de secundaria (62\%). Cerca de $94 \%$ del total de alumnos de ambos niveles educativos reportó tener acceso a Internet dentro de su casa (Tabla 1).

En general, el tiempo promedio de uso reportado fue de 6.2 horas diarias $(D E=4.1)$; al analizar estos tiempos por nivel educativo, se encontró que los estudiantes de ba- 
chillerato navegan un tiempo promedio mayor que los de secundaria. Aunque la mayoría de los participantes utiliza Internet menos de seis horas, en bachillerato casi 50\% registra un tiempo promedio mayor, a diferencia de los de secundaria, entre quienes $76.4 \%$ navegó menos de seis horas. Cerca de $80 \%$ de los estudiantes en ambos niveles educativos utiliza Internet de manera intermitente (Tabla 1).

Sobre las razones para conectarse a la red, 44\% del total de estudiantes declaró que la utiliza para revisar redes sociales y atender cuestiones académicas; el análisis por nivel educativo mostró porcentajes similares. El uso exclusivo para redes sociales fue mayor en alumnos de bachillerato que en los de secundaria; por el contrario, el uso de Internet con fines de entretenimiento (música, películas, juego en línea) fue mayor en alumnos de secundaria que en los de bachillerato (Tabla 1).

Resaltó que 33\% de los participantes presentó rasgos de impulsividad; variable que, por nivel educativo,

Tabla 1.

Características sociodemográficas de uso de Internet y personalidad por nivel educativo

\begin{tabular}{|c|c|c|c|c|}
\hline Característica & $\begin{array}{c}\text { Secundaria } \\
n_{1}=123\end{array}$ & $\begin{array}{c}\text { Bachillerato } \\
n_{2}=125\end{array}$ & $\begin{array}{c}\text { General } \\
n=248\end{array}$ & $p$ \\
\hline \multicolumn{5}{|l|}{$\operatorname{Sexo}^{a}$} \\
\hline Masculino & 41.5 & 46.4 & 44.0 & .5123 \\
\hline Femenino & 58.5 & 53.6 & 56.0 & .5123 \\
\hline Edad $^{\mathrm{b}}$ & 13.2(.9) & $16.3(1.0)$ & $14.8(1.8)$ & $.0000^{\star}$ \\
\hline \multicolumn{5}{|l|}{ Uso de Internet ${ }^{a}$} \\
\hline Problemático & 23.0 & 10.0 & 17.0 & $.009^{*}$ \\
\hline Normal & 77.0 & 90.0 & 83.0 & $.009^{*}$ \\
\hline \multicolumn{5}{|l|}{ Dispositivo de acceso a } \\
\hline Teléfono móvil & 61.8 & 81.6 & 72.0 & $.0009^{\star}$ \\
\hline Otros & 38.2 & 18.4 & 28.0 & $.0009^{*}$ \\
\hline \multicolumn{5}{|l|}{ Lugar de acceso $^{\text {a }}$} \\
\hline Casa & 93.5 & 94.4 & 94.0 & .9743 \\
\hline Otros & 6.5 & 5.6 & 6.0 & .9743 \\
\hline Tiempo promedio de uso ${ }^{b}$ & $5.0(3.8)$ & $7.3(4.0)$ & $6.2(4.1)$ & .0000 \\
\hline \multicolumn{5}{|l|}{ Tiempo de uso a } \\
\hline$>6 \mathrm{~h}$ & 23.6 & 48.8 & 36.0 & $.0001^{*}$ \\
\hline$<6 h$ & 76.4 & 51.2 & 64.0 & $.0001^{*}$ \\
\hline \multicolumn{5}{|l|}{ Horario de uso a } \\
\hline Continuo & 22.0 & 20.0 & 21.0 & .7059 \\
\hline Intermitente & 78.0 & 80.0 & 79.0 & .7059 \\
\hline \multicolumn{5}{|l|}{ Propósito del uso de Internet a } \\
\hline Redes sociales + académico & 40.7 & 48.0 & 44.0 & .2997 \\
\hline Redes sociales & 8.1 & 19.2 & 14.0 & $.0188^{\star}$ \\
\hline Académico & 6.5 & 1.6 & 4.0 & .1010 \\
\hline Entretenimiento & 44.7 & 31.2 & 38.0 & $.0391^{\star}$ \\
\hline \multicolumn{5}{|l|}{ Impulsividad a } \\
\hline Impulsivo & 27.0 & 39.0 & 33.0 & $.038^{*}$ \\
\hline No impulsivo & 73.0 & 61.0 & 67.0 & $.038^{*}$ \\
\hline
\end{tabular}

Fuente: Elaboración propia.

Nota:

${ }^{a}$ Los resultados se expresan en porcentajes. Para probar si los resultados entre los niveles educativos eran iguales o no, se realizó una prueba de Ji-Cuadrada.

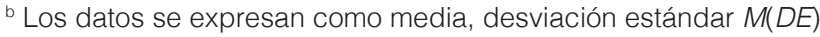

${ }^{*}$ Estadísticamente significativo $p<.05$ 
fue mayor en estudiantes de bachillerato (39\%) que de secundaria (27\%).

Debido a que sólo se encontró uso problemático, a partir de esta sección, el análisis se concentra en esta categoría. De acuerdo con la razón de momios $(O R)$, se demostró que los alumnos de secundaria tienen 2.5 veces más posibilidades de desarrollar un uso problemático de Internet. Los factores de exposición estadísticamente significativos para desarrollarlo fueron tiempo de uso y empleo exclusivo para redes sociales. Por lo anterior, el adolescente que pasa más de seis horas conectado a Internet tiene dos veces más posibilidades de desarrollar un uso problemático que quienes se conectan menos; el análisis de este resultado por nivel educativo mostró que los estudiantes de bachillerato tienen siete veces más posibilidades de desarrollar este uso y los de secundaria casi cuatro (Tabla 2).

El empleo exclusivo de Internet para conectarse a redes sociales resultó estadísticamente significativo en alumnos de bachillerato, quienes registraron más de cuatro veces la posibilidad de presentar un uso problemático que aquellos que se conectan con objetivos diversos (Tabla 2).

Las variables que no representaron un factor de riesgo estadísticamente significativo para el uso proble- mático de Internet, tanto de manera general como por nivel educativo, fueron sexo, tipo de dispositivo, lugar y horario de acceso, impulsividad y uso combinado de Internet - para conectarse a redes sociales y con fines académicos- o de manera exclusiva como entretenimiento (Tabla 2).

\section{DISCUSIÓN}

Es importante reconocer que el instrumento integrado que se utilizó en este trabajo es un instrumento de tamizaje y que, como lo recomendó el mismo Young (1998), hace falta un estudio clínico más profundo que valide el diagnóstico de Al. El criterio exclusivo del uso excesivo de la red no es suficiente para establecer un diagnóstico, toda vez que algunas personas se ven obligadas a pasar muchas horas conectadas debido a razones de trabajo o estudios, lo que no necesariamente las convertirá en adictas.

Los aspectos que se abordan en este trabajo son importantes porque, aunque la Al no tiene los efectos físicos asociados con la adicción a las sustancias psicoactivas, cada vez hay más estudios que establecen sus consecuencias negativas en la salud-como las derivadas de la privación de sueño (Young, 1998) y sus

Tabla 2.

Factores psicosociales asociados con el uso problemático de Internet por nivel educativo

\begin{tabular}{|c|c|c|c|c|c|c|}
\hline \multirow{2}{*}{ Característica } & \multicolumn{2}{|c|}{ Secundaria } & \multicolumn{2}{|c|}{ Bachillerato } & \multicolumn{2}{|c|}{ General } \\
\hline & OR & (IC 95\%) & OR & (IC 95\%) & OR & (IC 95\%) \\
\hline Nivel educativo secundaria & - & - & - & - & 2.539 & $(1.251,5.305)^{\star}$ \\
\hline Sexo masculino & 1.297 & $(.546,3.064)$ & .9891 & $(.295,3.240)$ & 1.122 & $(.567,2.210)$ \\
\hline Acceso a Internet a través del teléfono móvil & .944 & $(.396,2.301)$ & 2.915 & $(.465,66.010)$ & 0.9409 & $(.454,2.031)$ \\
\hline Acceso a Internet desde la casa & .877 & $(.174,6.641)$ & 1.919 & $(.104,35.526)$ & 1.305 & $(.318,8.834)$ \\
\hline Tiempo de uso mayor de 6 horas & 3.400 & $(1.342,8.614)^{\star}$ & 6.728 & $(1.583,46.490)^{*}$ & 2.659 & $(1.341,5.331)^{\star}$ \\
\hline Uso continuo & .961 & $(.345,2.677)$ & 1.926 & $(.541,6.856)$ & 1.267 & $(.5756,2.79)$ \\
\hline \multicolumn{7}{|l|}{ Propósito del uso de Internet } \\
\hline Redes sociales+ académico & .505 & $(.202,1.260)$ & .648 & $(.200,2.102)$ & 0.5265 & $(.258,1.073)$ \\
\hline Redes sociales & .648 & $(.200,2.102)$ & 4.476 & $(1.346,14.880)^{*}$ & 1.957 & $(.372,9.953)$ \\
\hline Entretenimiento & 1.915 & $(.816,4.492)$ & .369 & $(.078,1.749)$ & 1.349 & $(.6845,2.658)$ \\
\hline Impulsividad & 2.132 & $(.850,5.265)$ & 1.372 & $(.408,4.509)$ & 1.545 & $(.766,3.077)$ \\
\hline
\end{tabular}

Fuente: Elaboración propia.

Nota: *Estadísticamente significativo, el 1 no se incluye en el intervalo. 
repercusiones en el sistema inmune- y en las relaciones sociales, mismas que resultan afectadas por el debilitamiento de la comunicación familiar, la reducción de las redes sociales cara a cara y la aparición de síntomas de depresión.

Los resultados y los propios instrumentos utilizados en este estudio son de gran utilidad para ubicar qué proporción de la población adolescente estudiada enfrenta un uso problemático de Internet que puede transformarse en adicción; asimismo, sirven para entender cuáles son los factores que pueden estar influyendo en este hecho.

Como ya se estableció, se registró el uso problemático de Internet en $17 \%$ de la población participante: los estudiantes de secundaria fueron quienes presentaron un porcentaje mayor; este resultado supera lo reportado en otros estudios también realizados en México por Hernández Contreras et al. (2015), Méndez et al. (2014) y Beverido-Sustaeta et al. (2016). En independencia de estas discrepancias, el resultado obliga a formular interrogantes que deberán ser investigadas a mayor profundidad: ¿las diferencias encontradas se deben a un cambio en el nivel educativo?; cuando los estudiantes de secundaria lleguen al bachillerato ¿incrementarán su uso problemático y desarrollarán adicción a Internet?; ¿la falta de un incremento puede atribuirse al proceso de maduración de la corteza prefrontal o al hecho de que las exigencias académicas de este nivel educativo racionan el uso de la red?

El estudio permite establecer que el teléfono móvil es el principal medio que utilizan los adolescentes para conectarse a Internet, lo que puede explicarse porque se trata de estudiantes urbanos y por la facilidad de acceso que proporciona esta tecnología; este resultado coincide con lo reportado por Puerta-Cortés y Carbonell (2013) y Rial et al., (2014). También se encontraron coincidencias con otras investigaciones que muestran que la mayor parte de la población cuenta con el servicio de Internet en sus hogares (Castellana Rosell et al., 2007; Méndez et al., 2014; Puerta-Cortés \& Carbonell, 2013; Rial et al., 2014); sin embargo, es importante recordar que, por lo menos en este estudio, no se encontró evidencia estadísticamente significativa que permita concluir que se trata de un factor de exposición para desarrollar AI.

En contraste, los resultados apoyan una relación entre el tiempo de uso y el empleo problemático de Internet, por lo que se considera que a mayor tiempo de conexión, mayor probabilidad de riesgo; esto coincide con los resultados reportados por Muñoz-Rivas, Fernández y Gámez-Guadix (2010) en estudiantes españoles.

En relación con el uso que se le da a la Internet, el estudio establece que un factor de riesgo importante para el empleo problemático de la red, se registra cuando hay un uso exclusivo para redes sociales, lo que concuerda con el estudio de Puerta-Cortés y Carbonell (2013). Resulta preocupante este aspecto, pues el abuso de las redes sociales trae consigo la disminución del contacto personal, lo que puede dar como resultado el analfabetismo relacional, definido por Sánchez-Cabezudo (2011) como la ausencia de habilidades sociales básicas que posibilitan una relación positiva con el entorno y con los demás seres humanos; además, el uso de estas redes propicia el desarrollo de relaciones sociales ficticias (Echeburúa \& de Corral, 2010). Los resultados de esta investigación también coinciden con los de Muñoz-Rivas et al. (2010), al señalar que los estudiantes que usan Internet exclusivamente por razones académicas, no desarrollan un uso problemático.

No se encontró una relación significativa entre impulsividad y uso problemático de la red, lo que difiere de lo reportado en otras investigaciones que establecen que los individuos con mayor rasgo de impulsividad son más propensos a observar este tipo de uso (Gámez Guadix \& Villa George, 2015; Puerta-Cortés \& Carbonell, 2013; Zhang et al., 2015).

Una de las limitaciones del estudio consiste en que sólo se consideraron estudiantes de dos planteles educativos, por lo que los resultados no se pueden generalizar a la población de secundaria o bachilleres del estado o del país; sin embargo, el estudio contribuye a explorar y conocer algunas de las manifestaciones del problema en una ciudad capital como Xalapa, Veracruz.

En investigaciones futuras se debe considerar realizar estudios longitudinales para profundizar en el conocimiento del uso problemático de la Internet y la $\mathrm{Al}$ e incorporar otras variables como edad de inicio en el uso de la Internet, rendimiento académico, problemas de salud, consumo de drogas, síntomas de depresión y cambios comportamentales en diversas áreas.

Recapitulando, es posible establecer que, a pesar de las limitaciones, los hallazgos del estudio proporcionan una guía para futuras investigaciones que brinden información basada en evidencia a los psicólogos y psiquiatras que trabajan en la prevención, el diagnóstico y el tratamiento de los trastornos relacionados con el uso problemático de Internet en los adolescentes, con el fin de brindar una atención que evite la Al.

\section{FUENTES DE FINANCIAMIENTO}

No se obtuvieron fondos externos para la realización del presente estudio.

\section{CONFLICTOS DE INTERÉS}

No existen conflictos de interés. 


\section{AGRADECIMIENTOS}

Se agradece a las autoridades de la Escuela Secundaria y de Bachilleres Experimental de Xalapa, Enríquez, Veracruz, México.

\section{REFERENCIAS}

An, J., Sun, Y., Wan, Y., Chen, J., Wang, X., \& Tao, F. (2014). Associations between problematic Internet use and adolescents' physical and psychological symptoms: possible role of sleep quality. Journal of Addiction Medicine, 8(4), 282-287. doi: 10.1097/ADM.0000000000000026

American Psychiatric Association. (2014). Manual Diagnóstico y Estadístico de los Trastornos Mentales (5ta. ed.). Arlington, VA: American Psychiatric Publishing.

Beverido-Sustaeta, P., Camarena-Matús, D., Cruz-Juárez, A., \& Ortiz-León, M. (2016). Uso y abuso de la Internet en estudiantes de Ciencias de la Salud. Revista de Análisis Cuantitativo y Estadístico, 3(8), 36-42.

Caldevilla, D. (2010). Las redes sociales. Tipología, uso y consumo de las redes 2.0 en la sociedad digital actual. Documentación de las Ciencias de la Información, 33(1), 45-68.

Camelo, L., León, A., \& Salcedo, C. (2013). Adicción a Internet: aproximación a una perspectiva latinoamericana desde una revisión bibliográfica. Tercer Milenio. Periodismo y Comunicaciones, 18(25), 31-38.

Castellana Rosell, M., Sánchez-Carbonell, X., Graner Jordana, C., \& Beranuy Fargues, M. (2007). El adolescente ante las tecnologías de la informadión y la comunicación: Internet, móvil y videojuegos. Papeles del Psicólogo, 28(3), 196-204.

Centro Pedagógico Paideia (2016). Diferencias entre uso, abuso y adicción a las nuevas tecnologías. Recuperado de https://centropeda gogicopaideiaavila.wordpress.com/2016/02/02/diferencias-entre -uso-abuso-y-adiccion-a-las-nuevas-tecnologias/

Christakis, D. (2010). Internet addiction: a 21st century epidemic? BMC Medicine, 8(61), 1-3. doi: 10.1186/1741-7015-8-61

Cía, A. H. (2013). Las adicciones no relacionadas a sustancias (DSM-5, APA, 2013): un primer paso hacia la inclusión de las adicciones conductuales en las clasificaciones categoriales vigentes. Revista de Neuro-Psiquiatria, 76(4), 210-217. doi: 10.20453/rnp.v76i4.1169

Echeburúa, E., \& de Corral, P. (1994). Adicciones psicológicas: más allá de la metáfora. Clínica y Salud, 5(3), 251-258.

Echeburúa, E., \& de Corral, P. (2010). Adicción a las nuevas tecnologías y a las redes sociales en jóvenes: un nuevo reto. Adicciones, 22(2), 91-96. doi: 10.20882/adicciones.196

Gámez Guadix, M., \& Villa George, F. I. (2015). El modelo cognitivo-conductual de la adicción a Internet: el papel de la depresión y la impulsividad en adolescentes mexicanos. Psicología y Salud, 25(1), 111-122.

García del Castillo, J. A. (2013). Adicciones tecnológicas: el auge de las redes sociales. Salud y drogas, 13(1), 5-13.
Goldberg, I. (1995). Internet addiction disorder. Diagnostic criteria. Indiana: Internet Addiction Support Group. Recuperado de http:// www.iucf.indiana.edu/ brown/hyplan/addict.html

Greenfield, D. N. (1999). Virtual addiction: help for netheads, cyber freaks, and those who love them. Oakland: New Harbinger Publications.

Hernández Contreras, J., Ponce Rojo, A., \& Castañeda Barajas, A. (2015). Presencia del Trastorno de Adicción a Internet en estudiantes de un bachillerato en México. En XXVII AMIC Encuentro Nacional Querétaro 2015 (p. 15). Querétaro: Asociación Mexicana de investigadores de la comunicación.

Instituto Nacional de Estadística y Geografía. (2015). Encuesta Nacional sobre disponibilidad y uso de tecnologías de la información en los hogares. Recuperado de http://www.beta.inegi.org. mx/proyectos/enchogares/regulares/dutih/2015/default.html

Koran, L. M., Faber, R. J., Aboujaoude, E., Large, M. D., \& Serpe, R. T. (2006). Estimated prevalence of compulsive buying behavior in the United States. The American Journal of Psychiatry, 163(10), 1806-1812.

Lam-Figueroa, N., Contreras-Pulache, H., Mori-Quispe, E., Nizama-Valladolid, M., Gutiérrez, C., Hinostroza-Camposano, W., ... Hinostroza-Camposano, W. D. (2011). Adicción a Internet: desarrollo y validación de un instrumento en escolares adolescentes de Lima, Perú. Revista Peruana de Medicina Experimental y Salud Pública, 28(3), 462-469. doi: 10.1590/S172646342011000300009

Malloy-Diniz, L., De Paula, J. J., Vasconcelos, A. G., De Almondes, K. M., Pessoa, R., Faria, L., ... Mattos, P. (2015). Normative data of the Barratt Impulsiveness Scale 11 (BIS-11) for Brazilian adults. Revista Brasileira de Psiquiatria, 37(3), 245-248. doi: 10.1590/1516-4446-2014-1599

Martínez-Loredo, V., Fernández-Hermida, J. R., Fernández-Artamendi, S., Carballo, J. L., \& García-Rodríguez, O. (2015). Spanish adaptation and validation of the Barratt Impulsiveness Scale for early adolescents (BIS-11-A). International Journal of Clinical and Health Psychology, 15(3), 274-282. doi: 10.1016/j. ijchp.2015.07.002

Méndez, M. E., Zepeda Peña, H. H., \& Galván Álvarez, H. I. (2014). Usos de internet en estudiantes de educación secundaria de Puerto Vallarta. Revista Iberoamericana de Producción Académica y Gestión Educativa, 1(1), 1-16.

Muñoz-Rivas, M. J., Fernández, L., \& Gámez-Guadix, M. (2010). Analysis of the indicators of pathological Internet use in Spanish university students. The Spanish Journal of Psychology, 13(2), 697-707. doi: 10.1017/S1138741600002365

Muñoz-Rivas, M. J., Navarro, M. E., \& Ortega, N. (2003). Patrones de uso de Internet en población universitaria española. Adicciones, 15(2), 137-144. Recuperado de https://medes.com/ publication/10069

Oliva Delgado, A., García Hidalgo, M. V., Moreno Rodríguez, C., Jiménez García, L., Jiménez Iglesias, A., Antolín Suárez, L., ... Ramos Valverde, P. (2012). Uso y riesgo de adicciones a las nuevas tecnologías entre adolescentes y jóvenes andaluces 
(Aguaclara). Recuperado de http://passthrough.fw-notify.net/ download/510645/http://personal.us.es/oliva/libroadicciones.pdf

Ontiveros, E. (2015). Treinta años después. Evidencias e interrogantes. Telos: Cuadernos de Comunicación e Innovación, 100(Febrero-Mayo), 34-38. Recuperado de http://www.afi.es/EO/ notaprensa20150413a.pdf

Pedrero Pérez, E. J. (2009). Evaluación de la impulsividad funcional y disfuncional en adictos a sustancias mediante el Inventario de Dickman. Psicothema, 21(4), 585-591.

Pérez del Río, F. (2014). ¿El ocaso de la adicción a Internet?: reflexiones sobre el origen, desarrollo y declive de un trastorno. Revista Española de Drogodependencias, 39(2), 82-91.

Puerta-Cortés, D. X., \& Carbonell, X. (2013). Uso problemático de Internet en una muestra de estudiantes universitarios Colombianos. Avances en Psicologia Latinoamericana, 31(3), 620-631.

Rial, A., Gómez, P., Braña, T., \& Varela, J. (2014). Actitudes, percepciones y uso de Internet y las redes sociales entre los adoles- centes de la comunidad gallega (España). Anales de Psicologia, 30(2), 642-655. doi: 10.6018/analesps.30.2.159111

Sánchez-Cabezudo, S. S. (2011). Nuevas tendencias en el trabajo social con familias. Una propuesta para la práctica desde el empowerment. Madrid, España: Ed. Trotta.

Suriá, R. (2015). Disability in young people, increases the risk of excessive Internet use? Health and Addictions: Salud y Drogas, 15(1), 15-23.

Young, K. S. (1998). Internet addiction: the emergence of a new clinical disorder. CyberPsychology and Behavior, 1(3), 237-244. doi:10.1089/cpb.1998.1.237

Zhang, Y., Mei, S., Li, L., Chai, J., Li, J., \& Du, H. (2015). The relationship between impulsivity and Internet Addiction in Chinese college students: a moderated mediation analysis of meaning in life and self-esteem. Plos One, 10(7), e0131597. doi: 10.1371/ journal.pone.0131597 